Número DOI: 10.1590/permusi2015b3206

ARTIGO CIENTÍFICO

\title{
Contribuciones de Kepler a la continuidad y transformación de la tradición de la música de las esferas
}

\section{Kepler's contributions to the continuity and transformation of the tradition of the music of the spheres}

\author{
Johann F.W. Hasler ${ }^{1}$ \\ ${ }^{1}$ Facultad de Artes, Universidad de Antioquia UdeA. Calle 70 No. 52-21, Medellín, Colombia \\ johann.hasler@udea.edu.co
}

\section{Resumen:}

El presente artículo busca dar a conocer la importancia del trabajo de Johannes Kepler en la actualización, modificación, continuidad y transmisión posterior de las teorías de correspondencias entre planetas y alturas musicales en la tradición de la "Música de las Esferas", noción que para él tenía mucha importancia dentro de su concepción teológica del funcionamiento del cosmos. Lejos de desbancar esta antigua idea que en su origen funcionaba dentro del paradigma cosmológico geocéntrico, Kepler la adapta al sistema heliocéntrico copernicano y a sus propias propuestas de órbitas elípticas de los planetas, notando que estos nuevos modelos cosmológicos requieren de una nueva teoría de la música de las esferas: dado que se deduce que la velocidad de los planetas no es constante, esto tiene implicaciones importantes en los "tonos" (o dicho actualmente "alturas" o "notas") de cada planeta, que dejan de ser una altura única, como en los modelos de órbitas circulares, para convertirse en toda una gama musical. El desarrollo y evolución de la tradición de la Música de las Esferas es un largo capítulo en la historia de las ideas, de la ciencia y de la teoría musical (pues abarca desde Pitágoras hasta el presente), y hace parte de la llamada "música especulativa", que indaga las relaciones entre la teoría musical y sistemas considerados esotéricos o místicos. 
Este escrito aspira a contribuir, inicialmente a partir de la revisión de la literatura relevante, a este campo menos estudiado de la historia de la ciencia de la modernidad temprana desde el modelo medieval del quadrivium (aritmética, geometría, astronomía y música).

Palabras clave: Historia de la ciencia; historia de las ideas; Johannes Kepler; música de las esferas; música especulativa; música planetaria; órbitas elípticas.

\section{Abstract:}

This paper wishes to bring to a wider audience the importance of Johannes Kepler's efforts in the updating, modification, continuity and transmission of the theories of musical correspondences between the planets of the Solar System and the pitches of the musical scale. This tradition is known as "The Music of the Spheres", and its evolution and development constitutes a long chapter (from Pythagoras to the present day) in the history of ideas, science, and musical theory, through the area known as "speculative music", which researches on the connections between music (especially musical theory) and the occult, esoteric or mystical. As such, it warrants further research from the perspective of history of mentalities (understood in this case as a double take from both historical musiclogy and the history of science), and this is precisely the approach that his paper aims at.

Keywords: History of science; history of ideas; Johannes Kepler; music of the spheres; speculative music; planetary music; elliptical orbits.

Data de recebimento: 03/02/2014

Data de aprovação final: 20/09/2015 


\section{1 - Antecedentes y contexto}

El interés pitagórico en buscar correspondencias entre los sistemas naturales y las ciencias matemáticas se consolidó en la alta edad media a partir de los escritos de Boecio (480-524), de los cuales surgió la división de las artes liberales en el trivium y el quadrivium. Este último agrupaba las cuatro llamadas 'ciencias del número’: la aritmética (el número en sí mismo), la geometría (el número en el plano), la astronomía (el número en el espacio tridimensional) y la música (el número en el tiempo). Bien conocidos son los esfuerzos astronómicos que explicaban el funcionamiento de la mecánica celeste a través de la geometría de círculos, círculos dentro de círculos, y líneas de intersección. En este mismo espíritu integrador de todas las ciencias del quadrivium la teoría musical de escalas e intervalos también se utilizaba para entender el funcionamiento del cosmos, y desde Platón en adelante surgieron varias propuestas que asignaban cada una de las notas de la escala musical a las esferas celestes.

Johannes Kepler (1571-1630) fue uno de varios científicos importantes que vivió en el período de más intensa transformación que marcó el cambio entre la concepción medieval del conocimiento y aquella de la naciente modernidad temprana, que desembocaría en lo que conocemos como la revolución científica. Es interesante observar cómo Kepler quiso contribuir al antiguo debate de la música de las esferas, y continuó trabajando las correspondencias entre los planetas y las notas de la escala musical en su libro Harmonices Mundi (armonía de los mundos, de 1619), dedicando una buena parte de los libros III y V a la discusión armónica/musical de los movimientos celestes. En ese mismo escrito, Kepler combina además los dos principales métodos que hasta ese momento se habían aplicado para derivar tales correspondencias (uno por proporciones entre las órbitas planetarias, el otro por la velocidad de movimiento de los cuerpos celestes). Logra esta conjunción al extrapolar a la armonía de las esferas su otra innovación importante: las noción de las órbitas elípticas, y no circulares, de los planetas. En este sentido, Kepler mantiene la continuidad de la tradición de la armonía de las esferas aún cuando el cambio de paradigma que lo circundaba y que él mismo estaba ayudando a consolidar alejaba cada vez más lo exacto de lo expresivo - las ciencias de las artes. Al mismo tiempo, en un interés de mantener el vínculo entre ambos 
ámbitos del quehacer humano, Kepler adapta las nociones tradicionales de la música de las esferas a sus nuevas propuestas astronómicas, mostrándose así simultáneamente como continuador y reformador de la antigua tradición de la musica speculativa. Pero, ¿qué es la música especulativa?

\section{2 - La música especulativa como integración de lo supra-lunar con lo terrenal ${ }^{1}$}

El término "música especulativa", usado para referirse a todas esas tradiciones en la teoría musical que restan prioridad al análisis o estudio de obras ya compuestas, reemplazándolo por disquisiciones de cómo podría ser la música si resonara directamente con los ritmos de otros fenómenos naturales, o especulaciones sobre como debería ser para 'entonarse' mejor con el cosmos extra-humano, procede de la palabra latina speculum, que significa 'espejo'. Se llama así justamente porque su intención es hacer encajar la música de las esferas (la macrocósmica) con la música de las culturas humanas (la microcósmica), y la función de la teoría musical en esta ecuación es la de espejo (speculum), que permite que un nivel refleje al otro.

La música especulativa ha generado con el tiempo una teoría musical paralela a aquella más ampliamente conocida, la teoría musical que pudiéramos llamar 'mundana' o 'exotérica' que se enseña a los músicos durante su formación técnica, en tanto que la teoría musical esotérica o especulativa ha seguido siendo considerada como la 'auténtica' teoría musical en círculos esotéricos y ocultistas, pues refleja en nuestra música humana las leyes naturales de los mundos superiores, sean éstos físicos o espirituales, en tanto que la teoría musical exotérica o mundana se preocupa bien sea por generar normas para la creación de piezas musicales 'en este mundo', o de generar sistemas explicativos y de análisis de repertorios y usos musicales

\footnotetext{
${ }^{1}$ Para mayores detalles sobre este téma, consúltese mi artículo La Música Especulativa, en Ensayos, Historia y Teoría del arte. v.10, (2005) p.257-277.
} 
diversos, pero ya existentes. ${ }^{2}$ En suma, podemos afirmar que la teoría musical exotérica se preocupa por la música del mundo humano, en tanto que la teoría musical especulativa busca conexiones entre nuestro mundo y mundos no-humanos, sean físicos (p. ej. los astros), o espirituales (p. ej. las jerarquías espirituales o angélicas).

\section{1 - La teoría musical en la música especulativa}

Usualmente pensamos en la teoría musical como un sistema cerrado, relativamente abstracto (algunos la comparan con la matemática pura), ${ }^{3}$ y ciertamente auto-referencial, es decir, que hace referencia únicamente a relaciones musicales (intervalos, longitudes de duración de sonido, etc.) entre parámetros también estrictamente musicales (alturas, ritmos, etc), excluyendo de su sistema y sus teorizaciones toda relación con parámetros o sistemas extramusicales. En este sentido, la teoría musical pudiera interpretarse como un sistema teórico autocontenido y autoreferencial.

Esto es cierto para la teoría musical tradicional, que hemos llamado "exotérica", cuya tradición es mayormente descriptiva o prescriptiva (que busca analizar material musical ya existente, o dar normas para la creación de nuevo material dentro de unos procedimientos aceptados y altamente valorados), pero paralelamente a ésta se ha propuesto desde la antigüedad una teoría musical alternativa, que, en palabras del musicólogo Joscelyn Godwin "es la parte esotérica de la teoría musical, y como tal absorbe ampliamente ideas de [...] las ciencias ocultas" (GODWIN, 1995, p.4). ${ }^{4}$ Entre estas ciencias ocultas destaca por supuesto la astrología, que hizo parte de los estudios universitarios de astronomía hasta el siglo XVII (GENEVA, 1995 p.9-ss), y cuyo aparataje descriptivo y técnico hace uso de la matemática tal como lo hicieran desde la antigüedad los estudios pitagóricos de teoría musical. Estos últimos influyeron enormemente tanto en la teoría musical tradicional (exotérica) hasta la

\footnotetext{
${ }^{2}$ Sobre la diferenciación de los modelos especulativos, normativos y analíticos en la historia de la teoría musical de los griegos al presente, véase en extenso Christensen, (2002, p.1-26).

3 Véase a este respecto Risset, (2002, p.215-217).

${ }^{4}$ El original está en inglés, la traducción del pasaje es mía.
} 
edad media, como en la teoría musical especulativa (o esotérica), desde tiempos de Pitágoras hasta el presente. ${ }^{5}$

En efecto, la idea Pitagórica del número como llave para leer el universo e interpretar todas sus manifestaciones - incluyendo la música (GHYKA 1998) permitió que las herramientas matemáticas conocidas y desarrolladas por los seguidores ideológicos de Pitágoras (entre ellos varios teóricos de la música especulativa) ${ }^{6}$ fuesen aplicadas como puentes que permiten la traducción entre diversos sistemas, sobre todo entre aquellos que son usualmente descritos en términos matemáticos. De ahí que la astronomía y su aplicación a la vida humana, la astrología, ${ }^{7}$ hayan tenido una importante influencia en la configuración de la música especulativa.

Pero hay algo que quisiera dejar claro antes de continuar: como bien lo ha mencionado Godwin, "en su estado actual la música especulativa no es un cuerpo de conocimiento, ni nada que pueda ser aprendido y comunicado en un libro. Se trata más bien de una actitud mental.” (GODWIN, 1982, p.387). Por ello, sería desacertado afirmar que la teoría musical especulativa es un cuerpo teórico unificado, compartido y aceptado por todos sus estudiosos, y que lo que voy a exponer aquí es la historia de la teoría astrológica en la música especulativa, a la cual Kepler contribuye sustancialmente al adaptar la teoría de la música de las esferas al sistema heliocéntrico, y a su propuesta respecto a las órbitas elípticas de los planetas.

\footnotetext{
5 Dada su antigüedad y la suplantacion de sus bases epistemológicas por los paradigmas de ciencia mecanicista y materialista, sería excusable pensar que la teoría musical especulativa ya ha sido abandonada, y no ha habido desarrollos o investigaciones que continúen trabajando en este paradigma desde por lo menos la 'cientifización' de la teoría musical con Mersenne, Rameau, o Helmholtz entre los siglos XVII y XIX. Por el contrario, subsiten aún trabajos en este tipo de teoría, que desde los años setenta del siglo veinte ha estado en notable aumento. Véanse a este respecto Godwin (1982, p.373-389) y (1989) así como Hasler, (2005, p.257-277).

${ }^{6}$ Se puede acceder a una impresionante selección del trabajo de 48 diferentes autores en la tradición pitagórica en la música especulativa en Godwin (2009).

7 "La astrología es tanto el estudio de las manera cómo los objetos celestes son significantes para la vida en la Tierra, como las prácticas que resultan de esta idea”. (Campion, 2008, p.xi); Mi traducción del texto original en inglés.
} 


\subsection{Los planetas en la música especulativa}

Son tres los parámetros de los sistemas astrológicos occidentales que han atraído más la atención de los teóricos de la música especulativa: los planetas, los signos del zodiaco y sus respectivas casas astrológicas, y los aspectos angulares entre los planetas que se observan en una carta astral. Los tres son fundamentales a la hora de levantar una carta astrológica, y los tres han recibido asignaciones a alturas, tonalidades o intervalos a lo largo de la historia de la música especulativa. Pero el trabajo sobre los planetas ha siempre sido asociado a las alturas de la escala, por lo general heptáfona (dado que los planetas antiguos eran 7, igual que las notas de la escala heptáfona do, re, mi, fa sol, la si).

Es notoria la gran variedad de sistemas de atribución propuestos, y para el investigador de mentalidad moderna que busca una sola respuesta 'verdadera' resulta frustrante que los diversos sistemas históricos no solo no se validen entre sí - como esperaríamos en la ciencia positiva moderna - sino que por el contrario cada nueva teoría parece hacer una propuesta diferente que invalida o por lo menos pone en seria duda a las demás. La razón es el cambio histórico de tanto las teorías y modelos cosmológicos como de los sistemas y teorías musicales: así como han habido modelos cosmológicos diversos, de igual manera se han desarrollado teorías de asignación musical a los planetas basadas en ellos, y por lo tanto ampliamente divergentes.

Los antiguos conocían cinco de los planetas que actualmente definimos como tales, a saber: Mercurio, Venus, Marte, Júpiter y Saturno. Incluían también en esta lista al Sol y a la Luna, debido a que en cosmología geocéntrica - estándar científico hasta la propuesta Copernicana en el siglo XVI - todo lo que desde nuestra perspectiva terrestre se mueve alrededor de la bóveda celeste con relativa rapidez (incluyendo el Sol y la Luna, que de hecho son los más rápidos en cruzar la bóveda celeste) se entendía como 'planeta' (término derivado del griego planetes, que significa 'viajero', por el hecho de viajar por la bóveda celeste). 


\section{3 - Los tres modelos de asignación de alturas musicales a los planetas, anteriores a las propuestas de Kepler}

El modelo cosmológico más ampliamente aceptado antes de Copérnico era el de esferas concéntricas sobre la superficie de las cuales viajaban los planetas, a veces con rotaciones propias conocidas como epiciclos. Este modelo ya lo menciona Platón tanto en su Timeo como en las últimas páginas de La República (en el muy conocido 'mito de Er', en el libro X). La percepción de los sonidos cósmicos que producirían estas esferas, bien sea en estados alterados de conciencia (como en el caso del Er de Platón, o el de Escipión del sueño de Escipión de Cicerón) o la atribución y cálculo matematico-racional de estos sonidos a las esferas y sus movimientos, constituye la famosa idea de la "música de las esferas", y tiene una larga tradición en la historia de la teoría musical especulativa.

Tal vez porque los planetas suman justamente siete (el mismo número que las notas de la escala diatónica en el sistema griego y en el europeo subsiguiente que derivó de aquel), los teóricos de la música especulativa no pudieron dejar de ver la relación - al menos numérica - entre los planetas y las notas de la escala diatónica heptáfona, y en consecuencia asignaron a cada planeta una de las alturas de la escala diatónica. Pero no lo hicieron siguiendo siempre el mismo tipo de razonamiento.

El análisis de las teorías de asignación de planetas a clases de alturas ${ }^{8}$ demuestra que existen tres formas de deducir 'escalas planetarias', que el reconocido investigador en historia de la música especulativa Joscelyn Godwin ha catalogado como de 'Tipo A', 'Tipo B' y 'Tipo C' (GODWIN 1987, p.113-131). Las de tipo A están basadas en la relación proporcional de las distancias entre las órbitas de los planetas, las de tipo B están basadas en las velocidades

\footnotetext{
8 Este término técnico se refiere en teoría musical a todos los do-es, todos los re-es, todos los mí-es, y así sucesivamente, sin importar en qué octava aparezcan. Es decir, "clase de altura do" significa cualquier do, no únicamente el do central o el do de la octava superior o la inferior, o ningún do específico. Se trata de una generalización teórica por categorías de posición en la escala.
} 
aparentes de los astros en la bóveda celeste, y las de tipo C proponen una abstracción en la cual el universo conocido equivale a la gama musical conocida y en uso en la musica instrumentalis de Boecio, es decir en la música que hacemos las personas acá en la Tierra. Reseñaré a continuación estos sistemas, pero no en el mismo orden en que lo hace Godwin (primero A, luego B, luego C), pues deseo modificar el orden de la exposición por razones explicativas. Empezaré por lo tanto con el modelo B, pasando luego a los modelos A y C.

\subsection{Modelo B: las velocidades aparentes de los planetas determinan sus alturas musicales}

Los sistemas de asignación musical que Godwin llama de 'Tipo B' se basan en la relación proporcional de las velocidades de los planetas al cruzar la bóveda celeste, y no en la distancia entre ellos, como ocurre en las de tipo A, que discutiré más adelante. Esto aparentemente les da una base astronómica más sólida, porque, como comenta Godwin, "si bien las distancias entre los planetas han sido objeto de debate durante siglos, jamás ha habido ninguna duda en cuánto les toma darle una vuelta completa al zodiaco.” (GODWIN 1987, p.118) Pero el asunto no por ello resulta más sencillo, pues el cómputo de las velocidades de los astros variará dependiendo de si se considera que la tierra sea estática y que la última esfera, la de las estrellas fijas, se mueve lentamente a lo largo del año, o si, como también se proponía en algunos modelos cosmológicos griegos (como el de Anaximandro) se considera lo contrario, a saber que la tierra gira diariamente y que la esfera de las estrellas fijas es, como su nombre nos haría pensar, fija e inmóvil (DORCE-POLO 2006, p.9-29).

Dado que el sonido procede del movimiento (hoy en día lo entendemos como 'vibración'), y que mientras más rápido se mueva algo más agudo será el sonido que produzca, en los sistemas que usan la velocidad de translación de los cuerpos celestes sobre el horizonte los astros más rápidos producirán notas más agudas, en tanto que los más lentos producirán las notas graves. Si bien todos los sistemas de atribución basados en la velocidad planetaria comparten este razonamiento, las atribuciones que hacen entre planetas y alturas musicales

\footnotetext{
${ }^{9}$ Mi traducción del texto original en inglés.
} 
varían, pues algunos computan desde la tierra hacia arriba en las esferas, en tanto que otros computan desde las estrellas fijas hacia abajo, considerando al zodiaco como inmóvil, como ya se ha mencionado. Según varíe este punto de vista las velocidades se invierten, y por lo tanto también los sonidos que se deducen de ellas.

\subsection{Modelo A: las distancias entre las órbitas de los planetas determinan sus alturas musicales}

Las escalas planetarias que Godwin llama de 'Tipo A', por otro lado, no están basadas en la velocidad de los planetas, sino en la relación proporcional de las distancias de sus esferas u órbitas entre sí, tal como las calculaban los modelos astronómicos del momento. Por supuesto las asignaciones musicales que se derivan de ellos cambian con el tiempo, a la par que se desarrollan tanto los modelos astronómicos como de la teoría y la práctica musicales.

El razonamiento subyacente a este otro método de asignar alturas a los planetas es bastante sencillo: si asumimos que la octava musical (reducción teórica de todas las notas posibles en la teoría musical griega, como se ve en el ejemplo 1) corresponde al sistema solar y las estrellas fijas (el universo conocido en astronomía antigua), ello significa que las siete notas de la escala diatónica han de corresponder a los siete planetas (ejemplo 2).

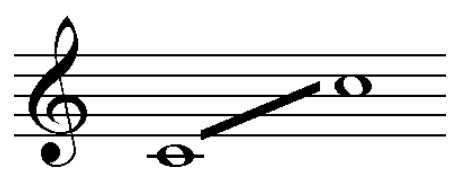

Ejemplo 1: La octava como universo musical (contiene todas las 'clases de alturas' de la teoría musical). ${ }^{10}$ Dado que es el universo musical teórico, según el paradigma especulativo deberá corresponder, por reflejo, también al universo macrocósmico, astronómico.

\footnotetext{
${ }^{10}$ Es decir, todos los do-es, todos los re-es, todos los mí-es, etc.
} 


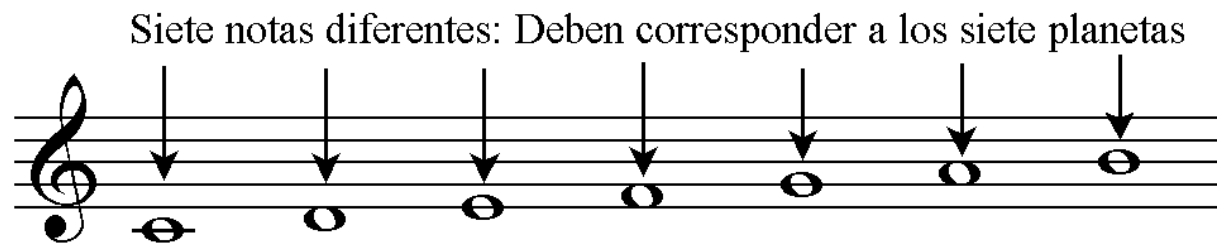

Ejemplo 2: Las siete diferentes clases de alturas de la octava musical en el sistema diatónico heptáfono, empezando aquí en $D O$. Nótese que son siete, tal como los planetas de los sistemas astronómicos antiguos.

Las distancias interválicas entre las notas de la octava en los sistemas musicales griegos únicamente son de tono o de semitono, siendo un tono entero equivalente a dos semitonos. Si podemos calcular la distancia entre un planeta y otro, y si esos cálculos nos muestran que entre las órbitas hay proporciones de 1:1 o de 2:1, la asignación de semitonos y tonos enteros a las distancias orbitales es básicamente directa, y se podría formar una escala teniendo en cuenta estas distancias.

Efectivamente, en los modelos cosmológicos antiguos, ya desde Pitágoras, se asumía que la distancia entre todos los planetas no era siempre la misma, y esta diferencia de distancias explicaba por deducciones geométricas las diferentes velocidades aparentes de los astros. Una de las ilustraciones más famosas de este tipo de especulaciones geométricas apareció en el Mysterium Cosmographicum de Johannes Kepler, en fecha tan tardía como 1596 (ejemplo $3)$. 


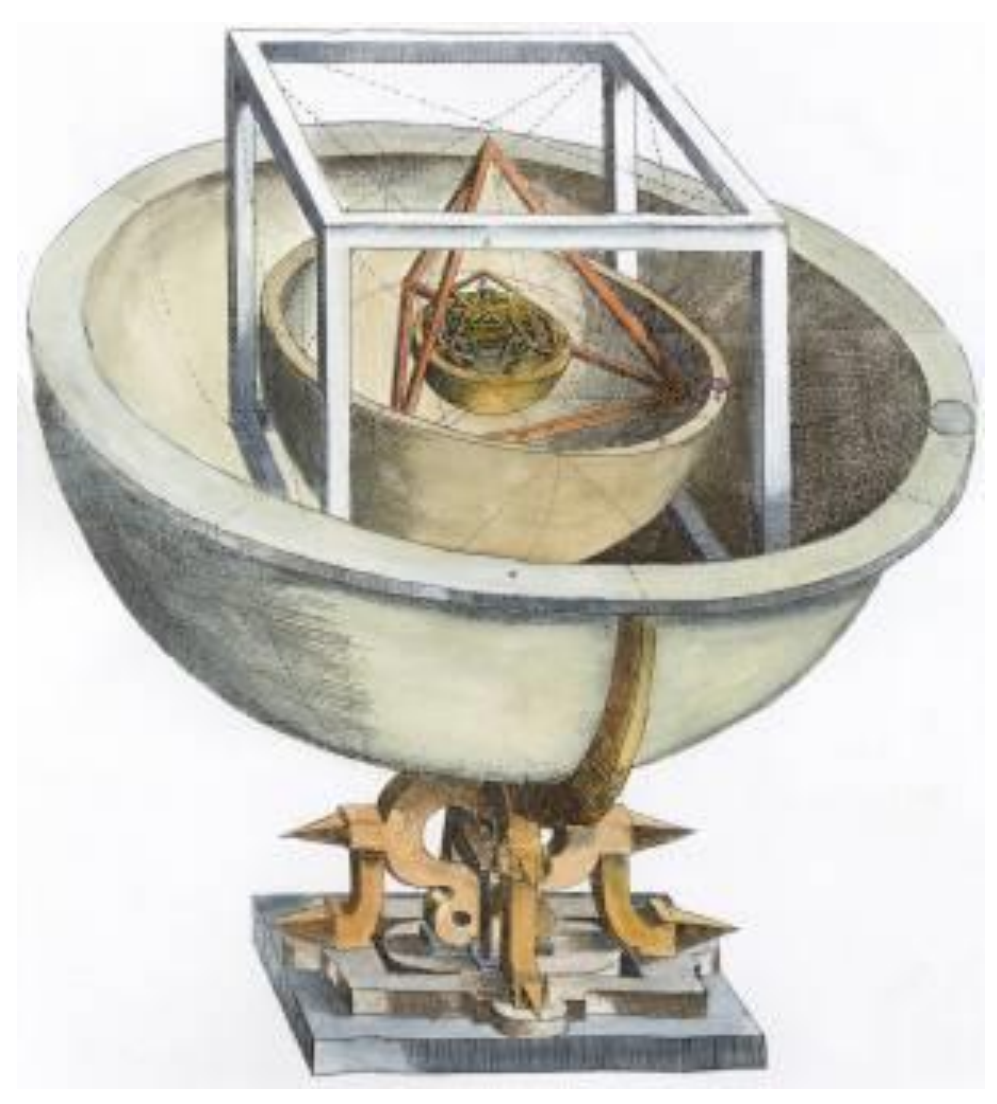

Ejemplo 3: Modelo cosmológico del Mysterium cosmographicum de Kepler (pub. 1596, 2a edición de 1621). Nótese que las órbitas o esferas de los planetas no son están todas a la misma distancia de separación (las de los planetas interiores están más cerca entre sí). En estas diferencias de separación entre las órbitas planetarias se basan los modelos de música de las esferas del 'Tipo A' (www.alchemywebsite.com/amcl_astronomical_material01.html).

En los modelos de atribución basados en las distancias entre las esferas, se cita la autoridad de Pitágoras, quien según los autores griegos calculó la distancia entre la Tierra y la Luna, y le asignó el intervalo del tono entero (GODWIN 1987, p.113). Comparando las distancias estimadas entre las demás esferas con la distancia entre la Tierra y la Luna, se puede deducir toda la escala heptáfona correspondiente a los siete planetas del sistema solar, y en algunos sistemas la distancia entre la esfera de Saturno y la de las estrellas fijas también. 


\subsection{Modelo C: 'el reflejo contrario': la teoría musical de la época en que se teoriza se extrapola a la música de las esferas}

El tercer tipo de atribución de planetas a alturas - los modelos de 'Tipo C de' Godwin (GODWIN 1987, p.126-131) es mucho más técnico, tanto así que considero que quedaría fuera de lugar en cualquier comunicación que no se presentara en un contexto especializado de musicología o teoría musical, por lo cual no entraré en detalles aquí. Baste mencionar que es el más teórico-musical y está menos basado en la observación de los fenómenos astronómicos que los anteriores modelos, y toma como base no notas específicas de una escala, o el orden de las notas en la misma, sino los puntos fijos de afinación de todos los modos y especies en la teoría musical griega (que también eran siete) ${ }^{11}$ y los relaciona a las distancias entre los nodos donde aparecen los armónicos naturales en la teoría acústica de cuerdas vibrantes de Pitágoras. Asume además como universo sonoro no la octava, sino toda la gama de alturas conocida y manejada por los instrumentos o la música vocal de cada época de la historia de la música, o inclusive teorizaciones que aún no se habían implementado en la música 'real' de la época de sus autores. ${ }^{12}$ Evidentemente, en la medida en que esta gama aumenta al aumentar las capacidades técnicas de los instrumentos y los desarrollos de la teoría que permiten nuevas propuestas en la práctica musical y generan nuevo repertorio (KRAEHENBUEHL y SCHMIDT 1962, p.32-65), también la gama del universo musical se hace cada vez más amplia, y con ello van cambiando las atribuciones a cada uno de los planetas que los modelos de este tipo proponen.

Como veremos más adelante, Kepler combina estos tres modelos en su novedosa propuesta.

\footnotetext{
11 Para ilustracion sobre teoría msuical griega, véanse Otto Gombosi (1951 p.20-26); Mathiesen, (2002, p.109-138) y Landels (1999, p.86-109).

12 Notable en este aspecto es la propuesta del parmesano Giorgio Anselmi (que no debe confundirse con su homónimo, el pintor veronés del siglo XVIII), quien en su libro De musica escrito en 1434 propone una gama de ocho octavas, cuando la música de su tiempo manejaba a lo más tres. Véase Godwin, Op. Cit., p.131.
} 


\section{4 - La contribución de Kepler a la adaptación de la idea de armonía musical de los planetas a las teorías y observaciones de Copérnico, Brahe, y las suyas propias}

Hasta la época de Kepler los tres modelos discutidos anteriormente constituían el estado del arte en cuanto a la atribución de alturas musicales a los planetas, con miras a presentar posibles propuestas musicales para la noción, a veces únicamente ideal o proporcional mas no necesariamente sonora, de la armonía de las esferas. Sin embargo, es apenas con Kepler cuando en este estado de cosas ocurre una revolución comparable a la que Galileo, Copérnico y el mismo Kepler causaron en la astronomía de su tiempo (y considero que no es coincidencia que la revolución científica de la primera modernidad se extendiera también a los aspectos más filosóficos o esotéricos de teorías especulativas, como es el caso en la de la música especulativa). En palabras de Daniel Walker, "en la larga tradición de la música de las esferas, las armonías celestes de Kepler son únicas en varios sentidos.” (WALKER 1967, p.228). Explicaré a continuación por qué.

Cuatro son las grandes contribuciones de Kepler a la actualización de la música de las esferas para ajustarla a los cambios en las concepciones astronómicas y las experiencias y teorías musicales de su tiempo. Se enumeran aquí sucintamente:

1. El Sol deja de sonar, en tanto que la Tierra adquiere una voz. Es la primera vez en la historia de la música de las esferas que esto sucede, y la razón es el cambio radical en el modelo cosmológico subyacente, que ha sido siempre la base de todas las teorías de la música de las esferas: al cambiar qué se mueve y qué permanece estacionario en un cierto modelo del Sistema Solar, cambia también el sonido o silencio que los cuerpos celestes produzcan, indistintamente si éste es real (acústico) o simbólico/metafórico (ideal). Por ello, en el sistema heliocéntrico que Kepler acoge, la Tierra pasa a moverse, y por lo tanto a generar sonido. 
2. La propuesta de Kepler es la primera en la historia de la música de las esferas que rechaza la entonación Pitagórica, prefiriendo la entonación justa, en fuerte discusión durante su tiempo. En esta entonación se encuentran más consonancias perfectas a la hora de superponer las voces en la escritura polifónica, en tanto que la entonación Pitagórica da unos intervalos más exactos y fáciles de cantar a la hora de hacer ciertos giros melódicos en la monofonía. Esto está en directa relación con los avances de la práctica musical polifónica en la época de Kepler.

3. La única manera de justificar matemáticamente el punto anterior es si se entienden las escalas como derivadas de figuras geométricas, y no de series numéricas. En consecuencia, Kepler rechaza las analogías numéricas utilizadas hasta su época por teóricos de la música de las esferas para describir las proporciones entre las órbitas de los planetas o sus velocidades, prefiriendo las analogías geométricas, por considerarlas menos abstractas y más basadas en las experiencias sensoriales de los seres humanos, las cuales, en su metafísica, son mejor indicio de la Mente Divina a la cual el ser humano debe intentar acercarse. Teológicamente esta fenomenología se explica así: si el ser humano fue creado a imagen y semejanza de Dios, y por lo tanto sus experiencias sensoriales son más adecuadas para dilucidar los misterios de la creación que las elucubraciones abstractas sin correspondencia directa con el mundo real.

4. Los cantos de cada uno de los planetas dejan de ser sobre una sola nota, como habían sido desde Platón hasta Zarlino (WALKER 1967, p.228) y en Kepler se transforman en escalas de diferentes longitudes para cada planeta, cuando en el pasado cada planeta hacía una sola nota de una única escala heptáfona (con prevalencia de la escala mayor en la mayoría de las propuestas históricas). Esto genera la posibilidad de polifonía melodico-armónica, como la que se da en la polifonía vocal de su tiempo, cuando en modelos anteriores se trataba más bien de acordes fijos cuyos componentes podían palidecer o aumentar en preponderancia y protagonismo de acuerdo a los diferentes momentos de posición planetaria, pero que permanecían siempre con una única nota por planeta. 
A continuación explicaré más extensamente cómo llega Kepler a conceptos tan revolucionarios en el ámbito de las músicas celestes.

\section{1) En cuanto a la asignación de un canto a la Tierra, y des-asignación de música al Sol:}

Al suscribirse Kepler al modelo heliocéntrico Copernicano, el punto central de la música de las esferas - así como del Sistema Solar - deja de ser la Tierra, y su lugar como punto inmóvil de referencia lo toma el Sol. Consecuentemente en la música de las esferas Kepleriana (dado que su propuesta es claramente especulativa, o sea que refleja en esta música ideal las realidades geométricas subyacentes del universo), el Sol deja de cantar en el concierto cósmico (pues los objetos estacionarios no producen sonido), en tanto que la Tierra adquiere voz cantante, al moverse alrededor del Sol.

Vemos aquí claramente cómo los modelos de música de las esferas dependen de los modelos cosmológicos que describen la configuración del Sistema Solar, y que al cambiar estos últimos, inevitablemente las especulaciones sobre cómo sería la música de los planetas antiguos necesariamente cambian también. Esta es una constante en la historia de la música de las esferas, que no se ve aquí por primera vez, dado que durante la antigüedad hubo también diversos modelos cosmológicos en competencia (DORCE 2006, p.9-30) que por espacio no podemos discutir aquí, pero que siempre resultaron también en modelos de música de las esferas con diferencias que reflejaban (recordemos que es música especulativa, es decir, que busca reflejar) en música los correspondientes cambios en los diversos modelos astronómicos del Sistema Solar.

\section{2) y 3) En cuanto al primer uso en la historia de la música de las esferas de la entonación justa en vez de la Pitagórica, y la utilización de figuras geométricas en vez de números naturales para la deducción de los intervalos en el nuevo sistema de entonación:}

Estas dos innovaciones, si bien diferenciables (listadas más arriba como 2 y 3), tienen la misma base matemático-acústica, por lo cual las trataré bajo el mismo encabezado. Daniel 
Walker nos informa que resulta evidente a través de su correspondencia que Kepler tenía y utilizaba un monocordio para sus experimentos acústico-matemáticos (WALKER 1967, p.239).

Y es posible que a través de la experimentación sobre ese monocordio comprobara empíricamente la consonancia de los intervalos de sexta y tercera, que los antiguos consideraban como disonantes. Kepler concluyó que el desprecio que profesaban los antiguos por la información que proveen los sentidos (en su visión fuertemente matemática y filosófica, intensamente anti-empiricista) los llevó a considerar que, matemáticamente, las terceras y las sextas deberían ser disonantes (pues sus teorizaciones aritméticas así lo indicaban), ignorando de este modo la percepción estética de las mismas, a través de la cual, según Kepler, resulta evidente que estos dos intervalos, cada vez más comunes en la música polifónica de su tiempo (por lo cual, presuponemos, Kepler estaría, si se quiere, 'acostumbrado' a ellos), se perciben como consonantes. Consigna esta opinión en su Harmonices Mundi:

"Los Pitagóricos estaban tan entregados a este filosofar a través de los números, que fracasaron en mantener el juicio de sus oídos, a pesar de que fue justamente por éste que llegaron a su filosofía en primer lugar. Así, definieron únicamente a través de sus números qué es y qué no es un intervalo melódico, qué es consonante y qué es disonante, violentando así el natural e instintivo juicio del oído.” (KEPLER 1940 [1619] vol. 6, p.99). ${ }^{13}$

Kepler profesaba esta preferencia epistemológica por los datos empíricos del oído (una posición en gran medida esteticista) ya desde su primera obra, 22 años anterior al Harmonices Mundi. Eso lo comenta más adelante en el mismo Harmonices, en un pasaje en el cual se refiere a esa obra, y en el cual se lee entre líneas que también en su tiempo existía una

${ }^{13}$ Citado por Walker en "Kepler's Celestial Music" (1967, p.235). Mi traducción del inglés, cotejando con el latín original para traducir con mayor claridad. 
polémica entre los defensores y los detractores de las terceras y las sextas bien sea como consonancias, o como disonancias:

"La evidencia presentada en mi libro Mysterium Cosmographicum será suficiente para proteger al sentido del oído contra las objeciones de los sofistas que se atreven a negar que se pueda tener fe en el oído cuando se trata de divisiones tan minúsculas [del monocordio] y tan sutiles distinciones de consonancias. Pues el lector verá que me he basado únicamente en el juicio del oído al establecer el número de las divisiones [es decir, de consonancias] aún en una época en la cual todavía estaba lidiando con encontrar las causas [es decir en su primera obra, el Mysterium Cosmographicum, de 1596], y que no hice entonces lo que los Antiguos hicieron: Pues ellos, habiendo avanzado un poco por el juicio del oído, pronto despreciaron esa guía, y continuaron el resto de su viaje siguiendo a la equivocada Razón, forzando así a sus oídos a abandonar el camino, ordenándoles, por así decirlo, a hacerse los sordos.” (KEPLER 1940 [1619] vol. 6 , p.19-20). ${ }^{14}$

Tenemos aquí un interesante ejemplo - aunque de nuevo no el único en la historia de la música de las esferas - donde resulta claro que el paradigma, pero sobre todo la práctica musical de la cual tiene experiencia un teórico (aunque sea únicamente como oyente), pueden ambos influir en los razonamientos que lo llevan a proponer cambios en una tradición intelectual que tal vez juzgamos, generalizando erróneamente, como intrínsecamente especulativa, filosófica, matemática, alejada de la realidad empírica, y no basada en la observación de las realidades musicales de su tiempo.

Pero, ¿cómo probará un astrónomo y matemático como Kepler que una nueva propuesta de entonación que él encuentra más adecuada para la música de su tiempo, pero que rechaza la tradición de números enteros de la entonación Pitagórica, es matemáticamente, además de estéticamente, preferible? ¿Cómo rechazar la prueba aritmética y aún así mantener una

\footnotetext{
${ }^{14}$ Citado por Walker en "Kepler's Celestial Music” (1967, p.239). La traducción del inglés es mía.
} 
prueba matemática sólida? Los pormenores de esta larga serie de demostraciones son tal vez la parte más árida y difícil de todo el Harmonices Mundi, y explicarlos en detalle aquí estaría, por su excesivo tecnicismo, más allá del ámbito de un escrito que se concentra en un aspecto puntual en la historia de las ideas. Bástenos apenas con informar que la solución que encuentra Kepler echa mano de la perspicacia geométrica que ya manifestó 22 años antes en su Mysterium Cosmographicum, donde soluciona el problema de la distancia entre las órbitas acudiendo a los cinco sólidos platónicos, intercalando cada uno entre las diversas esferas orbitales, solucionando así ese misterio de por qué las observaciones parecían indicar justamente esas distancias en las separaciones orbitales (véase el ejemplo 3, más arriba).

De manera similar, en Harmonices Mundi Kepler recurre a demostrar cómo la deducción de los intervalos a partir de cinco polígonos regulares que se encuentran repetidamente en la naturaleza - ya no a partir de una serie numérica abstracta - genera, efectivamente, el tipo de proporciones que se dan en la entonación justa, más adecuada para la música polifónica que la entonación Pitagórica, bella en melodías sueltas, pero con inaceptables disonancias a la hora de combinar varias voces simultáneamente.

\section{4) En cuanto a la asignación de escalas de diferente gama a cada planeta, en vez de una única nota a cada uno:}

Las elaboraciones geométricas de Kepler sobre los datos de Brahe acerca de la excentricidad en los movimientos observados de Marte lo llevaron a proponer órbitas elípticas para los planetas con el Sol como uno de los focos de la elipse, en vez de órbitas perfectamente circulares como se venía aceptando desde que Aristóteles determinara, siguiendo a Platón, que es el círculo la figura más simple y perfecta, y que la Naturaleza no podría estar diseñada sino con base en tan simple perfección. Según la segunda ley de movimiento planetario que Kepler formuló en 1609 en su libro Astronomia Nova, las órbitas elípticas generan diferencias tanto en la distancia entre el sol y los planetas en diferentes lugares de su órbita, como en las velocidades de los planetas en ciertos momentos de la misma (véase el ejemplo 4). 


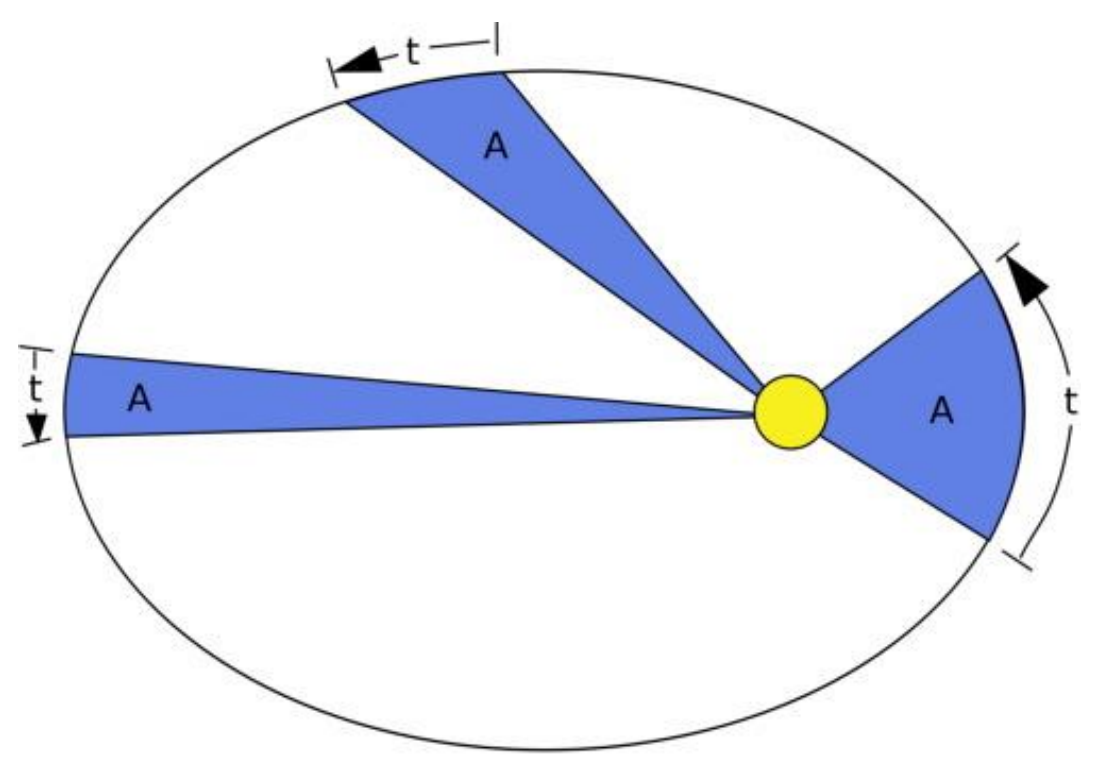

Ejemplo 4: Modelo orbital elíptico de Kepler donde queda claro cómo en el mismo tiempo

$(t)$ un planeta recorre un segmento de arco diferente (A), debido a que la diferencia de la distancia entre el planeta y el sol influye en la intensidad gravitacional que empuja al planeta en su movimiento. (www.astromia.com/universo/leyesuniverso.htm).

Esto implica que, siguiendo bien sea los modelos tipo A (velocidades orbitales=alturas) como los de tipo B (distancias desde el centro=alturas), en ambos modelos cada planeta no podrá dar una única altura, como había ocurrido hasta ese momento con los modelos cosmológicos basados en órbitas circulares (pues la distancia entre el Sol y los planetas sería siempre la misma), sino que daría una gama de alturas, correspondiendo la más grave al punto más alejado de la órbita al Sol (donde el planeta además se mueve más lentamente), en tanto que la altura más aguda corresponderá al punto más cercano de la órbita al Sol, donde el planeta se mueve más rápidamente. Dado que no todas las órbitas de los planetas son igualmente elípticas, y hay unas más excéntricas (es decir que se desvían más del círculo) que otras, el juego entre las diversas órbitas, de diferente excentricidad, con los planetas recorriéndolas a diferentes velocidades, siempre con un foco de la elipse en el Sol, pero con un punto diferente como el segundo foco (véase el ejemplo 5), todo ello generará inevitablemente un concierto de mucha más complejidad contrapuntística que lo que se da cuando todos los planetas viajan 
en órbitas perfectamente circulares y a velocidad uniforme. De este modo, deduce Kepler, el concierto de la armonía de las esferas bajo el nuevo modelo cosmológico Copernicano y de órbitas elípticas no puede sino llevar a una polifonía claramente comparable a la de la música vocal de su tiempo, y en este aspecto Kepler parece plegarse también a los modelos de atribución del tipo $\mathrm{C}: 15$

"Los movimientos de los cielos, por lo tanto, no son otra cosa sino un eterno concierto (racional, que no vocal) que tiende, a través de las disonancias, a través de lo que pudiéramos considerar como ciertas suspensiones o fórmulas cadenciales (por las cuales los hombres imitan esas disonancias naturales [de los cielos]), hacia cadencias definidas y prescritas, con cada acorde con seis términos (es decir a seis voces), y que a través de esas marcas [es decir las cadencias] se distingue y articula la inmensidad del tiempo; de tal manera que ya no es de maravillarse que finalmente esta manera de cantar a varias partes, desconocida para los antiguos, haya sido inventada por el Hombre, el Simio de su Creador; de tal manera que a través de la sinfonía artificial de varias voces pueda él [el hombre] representar, en la breve duración de una hora, la perpetuidad de la duración total del cosmos, y de este modo pueda percibir en cierto grado la satisfacción de Dios el Creador con Sus propias obras, con el más intensamente dulce placer obtenido de su Música, que imita a Dios. (KEPLER 1940 [1619] vol. 6, p.328)."16

\footnotetext{
15 Recordemos que en los modelos del tipo $\mathrm{C}$ el reflejo es al contrario de los otros dos modelos, pues no se considera problemático que la teoría cosmológica se deduzca reflejando el comportamiento de la música humana acá en la Tierra.

${ }^{16}$ Citado por Walker en "Kepler's Celestial Music" (1967, p.250). La traducción del inglés es mía.
} 


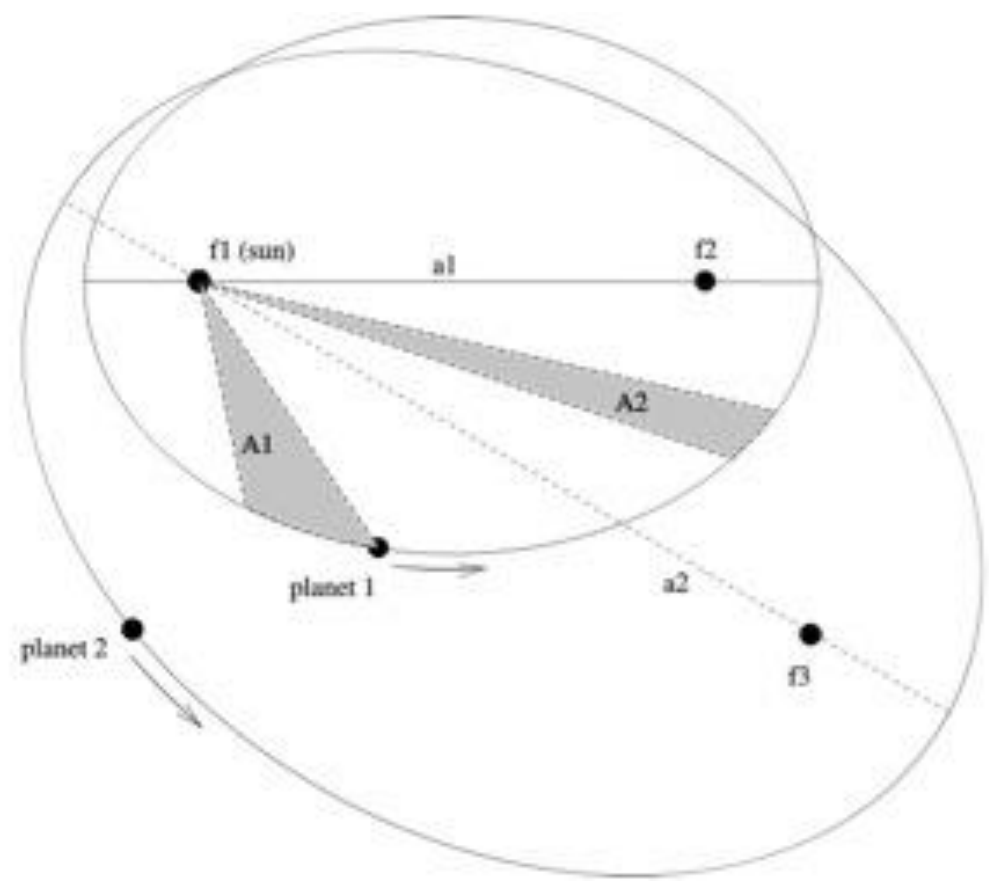

Ejemplo 5: Esquema de las complejas variaciones de distancias y velocidades que se dan cuando dos órbitas elípticas presentan diferentes focos secundarios (f2 y f3) además de uno compartido - el sol - debido a sus diferentes excentricidades. https://upload.wikimedia.org/wikipedia/commons/archive/9/98/20111123142822\%21Keple r_laws_diagram.svg)

\section{5 - Conclusiones}

De esta forma, en la propuesta orbital que Kepler hace en Astronomia Nova (1609), y que luego retoma musicalmente en Harmonices Mundi (1619), encontramos la más importante transformación que hace dentro de la continuidad de la tradición de la música de las esferas: Kepler conoce todos los modelos anteriores de atribución de alturas a los planetas, pero en el suyo propio, debido a los datos astronómicos y nuevas teorías cosmológicas (vg. el Copernicanismo y las órbitas elípticas), decide no simplemente rechazarlos de plano al no coincidir con los datos cosmológicos observados - como harían otros contemporáneos suyos como Marin Mersenne, quien, inversamente pero por razones comparables rechazó tanto el heliocentrismo como las órbitas elípticas (FABBRI 2003, p.54) - sino que los combina, 
generando si se quiere un 'modelo $\mathrm{A}+\mathrm{B}+\mathrm{C}$ ', que pudiéramos expresar esquemáticamente como 'alturas=distancia al sol y velocidad orbital aparente'. Es más, dado que para Kepler resulta lógico que el concierto celeste sea polifónico y a seis voces (una voz por cada planeta, sin tener en cuenta ni al Sol ni a la Luna), como lo era la elaborada música contrapuntísitca de su tiempo, también hay mucho del 'modelo C' en su razonamiento, como queda demostrado por la cita extensa presentada más arriba: Kepler deduce que si la velocidad de translación de los planetas ya no es constante, pues igualmente ya no producirán un solo sonido cada uno - lo cual era la norma hasta ese momento en música de las esferas - sino que a cada planeta le correspondería una gama entera de sonidos, el más agudo procedente de su perihelio (el punto más cercano de la órbita al sol, donde el planeta viaja más rápido) y el más grave a su afelio (el punto más lejano de su órbita, donde viaja más lentamente).

Esta última gran innovación de Kepler a la tradición de la música de las esferas, a saber la combinación de todos los modelos paradigmáticos para atribución de alturas a los planetas del sistema solar (tratados en este artículo como sistemas de tipo A, B y C, siguiendo la nomenclatura utilizada por Godwin) es la contribución más notable de Kepler a esta tradición, y de ella podemos deducir su respeto y su fe en esta antigua idea, al punto de querer mantenerla y actualizarla para que pudiera seguir vigente aún en el contexto de los nuevos modelos cosmológicos. Kepler tiene la esperanza de que la tradición de la música de las esferas evolucione, en vez de convertirse en obsoleta, como ocurriría pocas décadas después de su muerte, a manos de los representantes de la nueva ciencia positivista.

En efecto, para el siglo XVIII ya ningún científico tomaba en serio la idea de que los planetas, en sus movimientos, llevaran una cierta armonía proporcional comparable a la de la música de los seres humanos, y la idea de la música de las esferas migró del campo científico y filosófico al metafísico, místico o puramente esotérico (Godwin 1986), tal como podemos comprobar por los antecedentes y credenciales de los autores más sobresalientes que trabajaron este tema a partir de ese momento de la historia: entre los autores místicos y esotéricos Godwin nos presenta a Fabre d'Olivet, Saint-Yves D'Alveydre, Rudolf Steiner y George Ivanovich Gurdjieff; en tanto que a nivel científico apenas hasta el siglo XX empieza 
a resurgir la idea en el ámbito de la ciencia, pero únicamente en los gremios un tanto 'paralelos' y ‘underground' de la nueva 'ciencia armónica' que surge en los países germanos, en un programa neo-pitagórico de volver a buscar la unidad de los diversos aspectos del universo a través de la matemática y la geometría (Godwin 1982, 1989 y 2009).

En este respecto deben mencionarse los diversos trabajos de Hans Kayser (1891-1964), Hans Erhard Lauer (1899-1979), Marius Schneider (1902-1982), Rudolf Haase (n. 1920), y más recientemente Hans Cousto (n. 1948) y Hartmut Warm (n. 1956), todos representantes de esta nueva tendencia que continúa, en su espíritu original, las búsquedas mistico-cientificoespeculativas que Kepler presentara a lo largo de toda su obra, pero especialmente en su primer libro, Mysterium Cosmographicum (1596) y en la culminación teórica de sus especulaciones musicales, geométricas y proporcionales, Harmonices Mundi, escrita 22 años después.

\section{Referencias}

1. CAMPION, N. (2008). A History of Western Astrology. Volume 1, The Ancient World. Londres: Continuum.

2. CHRISTENSEN, Th., (ed.) (2002). The Cambridge History of Western Music Theory. Cambridge: Cambridge University Press.

3. COUSTO, H. (2000). The Cosmic Octave: Origin of Harmony. Mendocino, California, EUA: LifeRhythm.

4. DORCE POLO, C. (2006). Ptolomeo, el astrónomo de los círculos. Madrid: Nivola Ediciones.

5. FABBRI, N. (2003). Cosmologia e armonia in Kepler e Mersenne: contrappunto a due voci sul tema dell'harmonice mundi. Florencia: Leo S. Olschki.

6. GENEVA, A. (1995). Astrology and the Seventeenth Century Mind: William Lilly and the Language of the Stars. Manchester: Manchester University Press.

7. GHYKA, M. (1998). Filosofía y mística del número. Barcelona: Apóstrofe. 
8. GODWIN, J. (1982). "The Revival of Speculative Music". Musical Quarterly. v. $67 \# 3$, p.373-389.

9. EUA: Arkana (Penguin).

(1986). Music, Mysticism and Magic: A Sourcebook. Nueva York, 10. (1987). Harmonies of Heaven and Earth: the Spiritual Dimensions of Music from Antiquity to the Avant-garde. Rochester, Vermont, EUA: Inner Traditions.

11. (1989). Cosmic Music: Musical Keys to the Interpretation of Reality. Rochester, Vermont, EUA: Inner Traditions.

12. (2009). Armonía de las esferas: un libro de consulta sobre la tradición pitagórica en la música. Girona: Atalanta.

13. GOMBOSI, O. (1951). "Key, Mode, Species”. Journal of the American Musicological Society. v. 4\#1, p.20-26.

14. HAASE, R. (1989). "The Sequel to Kepler's World Harmony." En Godwin, J. (ed.) Cosmic Music: Musical Keys to the Interpretation of Reality. Rochester, Vermont, EUA: Inner Traditions, p. 131-144.

15. HAASE, R. (1989). "Kepler's World Harmony and Its Significance Today." En En Godwin, J. (ed.) Cosmic Music: Musical Keys to the Interpretation of Reality. Rochester, Vermont, EUA: Inner Traditions, p. 111-130.

16. HAASE, R. (1989). "Harmonics and Sacred Tradition." En En Godwin, J. (ed.) Cosmic Music: Musical Keys to the Interpretation of Reality. Rochester, Vermont, EUA: Inner Traditions, 1989, p. 91-110.

17. HASLER, J.F.W. (2005). "La música especulativa”. Ensayos, historia y teoría del arte. v.10, p. 257-277.

18. KRAEHENBUEHL, D.; SCHMIDT, CH. (1962). "On the Development of Musical Systems". Journal of Music Theory. v.6:1, p.32-65.

19. KAYSER, Hans. Textbook of Harmonics. Editado por Joscelyn Godwin. 2 vols. Idlyllwild, California, EUA: Sacred Science Institute, 2006.

20. KEPLER, J. (1940). "Harmonices Mundi” [1619] en Caspar, M. (ed.), volumen 6 de Gesamte Werke. Munich: Beck.

21. KEPLER, J. (2005) "Briefe 1607-1611” en Schenkel P.M. (ed.), volumen 16 de Gesamte Werke. Munich: Bayerische Akademie der Wissenschaften. 
22. KEPLER, J. (1997). The Harmony of the World. Translated into English with an Introduction and Notes by E.J. Aiton, A.M. Duncan, \& J.V. Field. Philadelphia, Pennsylvania, EUA: American Philosophical Society.

23. LANDELS, J.G. (1999). Music in Ancient Greece \& Rome. Abingdon, Inglaterra: Routledge.

24. LAUER, H. E. (1989) "The Evolution of Music through Changes in Tone-Systems." En Godwin, J. (ed.) Cosmic Music: Musical Keys to the Interpretation of Reality. Rochester, Vermont, EUA: Inner Traditions, p. 150-167.

25. MATHIESEN, TH. (2002). "Greek music theory". En The Cambridge History of Western Music Theory, editado por Thomas Christensen. Cambridge: Cambridge University Press, , p. 109-138.

26. RISSET, J.C. (2002). "Mathematics and Musical Theory". En Mathematics and Music: A Diderot Mathematical Forum,. Editado por Gerard Assayag, Hans Georg Feichtinger y José Francisco Rodrigues. Berlin: Springer, p. 215-217.

27. WALKER, D.P. (1967) "Kepler's Celestial Music". Journal of the Warburg and Courtauld Institutes v.30, p. 228-250.

28. WARM, H. (2010) Signatures of the Celestial Spheres: Discovering Order in the Solar System. Forest Row, Inglaterra: Sophia Books.

29. WARRWAIN, F. (1942). Essai sur l'Harmonices Mundi ou Musique du Monde de Johann Kepler. Paris: Hermann \& cie.

Nota sobre el autor

Johann Hasler es profesor del Departamento de Música de la Universidad de Antioquia en Medellín (Colombia), y director del grupo de investigación "Artes y Modelos de Pensamiento" de la Facultad de Artes, en el cual se indagan, entre otros temas, la historia de la teoría musical y su relación con otros modelos de pensamiento, tales como la astronomía o el esoterismo en todas sus manifestaciones (incluyendo platonismo musical, neopitagorismo, 'ciencia armónica', astrología y alquimia). Es también director de la línea de investigación "esoterismos y otras religiosidades mágicas" del grupo de investigación "Religión, Cultura y Sociedad”, de la misma universidad. johann.hasler@udea.edu.co; artesymodelosdepensamiento@gmail.com. 